\title{
Some Properties on Divine Kaehlerian Manifold
}

\author{
Kailash Chandra Patwel ${ }^{{ }^{*}}$ \\ ${ }^{1}$ Department of Mathematics, H.N.B. Garhwal University Campus Badshahithaul, Tehri Garhwal, Uttarakhand, \\ India
}

*Corresponding Author Email id: kcpetwal@gmail.com

Received: 11.04.2021; Revised: 16.06.2021; Accepted: 19.06.2021

(C)Society for Himalayan Action Research and Development

\begin{abstract}
Fibonacci sequence and the divine ratio are intimately inter-connected. In the Fibonacci sequence each number is the sum of previous two consecutive numbers and the ratio of any two consecutive numbers reflects the approximate value of divine ratio. The relationship between divine ratio and Fibonacci series is well express in divergent faunal anatomy and floral as well as their morphology. The present article is intended to study the properties of divine Kaehlerian manifold in terms of Fibonacci sequence, trace \& eigen values of divine structure including its almost complex structures. Some properties of induced structures, theorems and propositions related to it have also been studied.
\end{abstract}

M.S.C. (2010): 53B20,53C15

Keywords: Divine $\bullet$ Fibonacci $\bullet$ Kaehlerian $\bullet$ Manifold $\bullet$ Sequence $\bullet$ Tensor

\section{Introduction}

Our nature is really beautiful adding mathematics with divergent branches of science. The Fibonacci has an apparent contact with the Statistics; Operational Research and Computational mathematics covering divergent geometric topics for example divine section etc. The ideas of Fibonacci number are widely relevant to growth of every living thing, involving a cell, from a wheat grain to a bee's hive. The universe may be unsettled and uncertain, but it also an overmuch unified physical region bound by mathematics laws. From the primitive times Fibonacci numbers attracts mathematics for their unique beauty and abounded notions possessing a unique feature with their notions in divergent region of science unrelated to mathematics (Sarma and Bhuyar, 2018). Golden ratio or golden section or divine proportion is those number which is approximately equal to 1.618 this number is also called an irrational mathematical constant 'phi' and is denoted the letter $\varphi$ from the Greek alphabet. In the easiest form Divine ratio is the division of a line into an exclusive ratio producing an artistically attractive fraction, keeping a pathetic usage in architecture and art. Divine ratios were also reported to be present in divergent plant anatomy as well as human body parts (http://en.).

A Kaehler manifold as well as complex manifold (Y) that is closed with 2-form and has a hermitian matrix h. In greater detail, at each point of complex manifold, $\mathrm{h}$ gives a positive definite hermitian form on the tangent space and the $\mathbf{w} 2$ form such that:

$w(u, v)=\operatorname{Re} h(i u, v)=\operatorname{im} h(u, v)$

For vector tangent $\mathbf{u}$ and $\mathbf{v}$ (where $i$ is the complex number $\sqrt{-1}$ ) for a Kaehler manifold, the Kaehler form $\mathbf{w}$ is real and closed to 1,1 -form. A Kaehler manifold can also be viewed as a Riemannian manifold (R) with Riemannian metric $g$ such that: $g(u, v)=\operatorname{Re} h(u, v)$ 
A Kaehler manifold is a hermitian manifold of complex dimension $\mathrm{n}$, such that for every point $\mathrm{Q}$ of complex manifold, there is a holomorphic co-ordinate chart around $\mathrm{r}$, in which the metric on $C^{n}$ is represented in these co-ordinates as:

$h_{a b}=\left(\frac{\partial}{\partial z_{a}}, \frac{\partial}{\partial z_{b}}\right) h_{a b}=\left(\frac{\partial}{\partial z_{a}}, \frac{\partial}{\partial z_{b}}\right)$, then $h_{a b}=\delta_{a b}+0\left(\|z\|^{2}\right) h_{a b}=\delta_{a b}+0\left(\|z\|^{2}\right)$

For all $\mathrm{a}, \mathrm{b}$ lies from $1,2,3, \ldots \ldots . \mathrm{n}$.

The 2-form is closed in this case, and thus determines the Kaehler class in De-Rham cohomology $H^{2}(Y, R)$. A Kaehler manifold is an even-dimensional Riemann manifold $\mathrm{X}$ with a holonomy group contained in the unitary group $u(n)$. In addition, at each point on the tangent space of complex manifold (Y), there is a complex structure $\mathrm{J}$ (which is a real linear map from $T_{y}$ and $J^{2}$ is equal to negative one) and maintains the metric $g$. As a result, $\mathrm{J}$ preserves parallel transport and the alternating two-form is as follows:

$$
w(Y, X)=g(J Y, X)
$$

Which shows that, if $\mathbf{w}$ is closed then $g$ is a Kaehler metric in Kaehler manifold $N$.

\section{Divine Kaehlerian Manifold}

In this part we prescribe the structure of polynomial in a n-dimensional Kaehlerian manifold. The structure represented by $(\widetilde{N}, \widetilde{h})$ called divine structure, which determine by $1,1-$ type tensor field $\widetilde{Q}$. It is sufficient for the equation

$$
\widetilde{Q}^{2}=\tilde{Q}+I
$$

where, identity operator (I) is on the Lie derivative $\eta(\widetilde{N})$ of vector fields N. Here, the hermitian matrix $\breve{h}$ in $\tilde{Q}$ is applicable, if the similarity

$$
\widetilde{h}(\tilde{Q}(W), X)=\tilde{h}(W, \tilde{Q}(X))
$$

It is sufficient, for every vector field of tangent $W, X \in \eta(\widetilde{N})$.

Note: For a divine structure $\breve{Q}$, Kaehlerian manifold $(\widetilde{N}, \tilde{h})$ is equivalent with

$$
\tilde{h}(\tilde{Q}(W), \tilde{Q}(X))=h(W, \tilde{Q}(X))+\tilde{h}(W, X)
$$

$\forall$ tangent vector fields $U, V \in \Pi(\widetilde{N})$.

Theorem 2.1: A divine Kaehlerian manifold $(\widetilde{N}, \widetilde{h}, \tilde{Q})$ has the property

$\tilde{Q}^{m}=\varphi_{m} \tilde{Q}+\varphi_{m-1} I$

$\forall$ integer number $m>0$, where $\left(\varphi_{m}\right)_{m}$ is the Fibonacci sequence.

Proof: If we suppose

and

$$
\begin{aligned}
\widetilde{Q}^{n} & =\varphi_{n} \tilde{Q}+\varphi_{n-1} I \quad(n>0), \text { we get } \\
\tilde{Q}^{2} & =\tilde{Q}+I \widetilde{Q}^{2}=\tilde{Q}+I ; \tilde{Q}^{3}=2 \tilde{Q}+I \\
\widetilde{Q}^{4} & =3 \tilde{Q}+\widetilde{Q}^{n+1}=\varphi_{n} \widetilde{Q}^{2}+\varphi_{n-1} \widetilde{Q} \\
& =\left(\varphi_{n}+\varphi_{n-1}\right) \tilde{Q}+\varphi_{n} I
\end{aligned}
$$

Hence the result. 
Remark: A Kaehlerian manifold $(\widetilde{N}, \tilde{h})$ with a divine manifold $\widetilde{Q}$ is signify by divine Kaehlerian structure and $(\tilde{h}, \tilde{Q})$ itself a divine Kaehlerian structure on N.

Theorem 2.2 The trace and Eigen values of the divine structure $\tilde{Q}$ defined in an $n$-dimensional Kaehlerian manifold $(\tilde{h}, \tilde{Q})$ are the divine ratio trace $\left(\widetilde{Q}^{2}\right)=\operatorname{trace}(Q)+n$ and the eigen values are $\Psi$ and $(\Psi-1)$

Proof: Let $\left\{f_{1}, f_{2} \ldots \ldots \ldots \ldots f_{n}\right\}$ be an orthonormal basis of the tangent space $T_{y} \widetilde{N}$ on each point of $y \in \widetilde{N}$. Now, from eq. (2.1), we get

$$
\tilde{h}\left(\tilde{Q}^{2} f_{i}, f_{i}\right)=\tilde{h}\left(\tilde{Q} f_{i}, f_{i}\right)+\tilde{h}\left(f_{i}, f_{i}\right)
$$

Summing it by $i$, we have

$$
\operatorname{trace}\left(\tilde{Q}^{2}\right)=\operatorname{trace}(Q)+n
$$

Further, if $\lambda$ is an eigen value of the divine structure $\widetilde{Q}$ on $T_{y} \widetilde{N}(\forall y \in \widetilde{N})$ then $\widetilde{Q} X=\lambda X$ for all vector fields of tangent $X \in T_{y} \widetilde{N}$. At each point of $y \in \widetilde{N}$, we get $\lambda^{2}=\lambda+I$,

which follows that eigen values of $\tilde{Q}$ are divine ratio $\lambda_{1}=\Psi$ and $\lambda_{2}=I-\Psi$.

Remark: If Kaehlerian manifold $\widetilde{N}$ is an n-dimensional completed with a positive definite Kaehlerian hermitian matrix $\widetilde{h}$ and suppose that a non-trivial field of tensor $\mathrm{K}$ of $1,1-$ type such that $K^{2}=I$ and $\tilde{h}(K u, K v)=\tilde{h}(u, v)$ for all vector fields $u, v \in \eta(\widetilde{N})$, then $\mathrm{K}$ is known as an almost product structure and $(\widetilde{N}, \widetilde{h}, \widetilde{K})$ represent as an almost product Kaehlerian manifold.

Proposition 2.3 Each almost product structure $K$ in an $n$-dimensional Kaehlerian manifold $(\widetilde{N}, \tilde{h})$ induced to divine structures on $(\widetilde{N}, \tilde{h})$ as:

$$
\widetilde{Q}_{1}=\frac{l+\sqrt{5} K}{2}, \widetilde{Q}_{2}=\frac{l-\sqrt{5} K}{2}
$$

vice-versa. Also, the divine structure $\tilde{Q}$ prescribed on a Kaehlerian manifold $(\widetilde{N}, \tilde{h})$ induced with an almost product structure of this manifold.

Proof: Let almost product structure K prescribed in an n-dimensional Kaehlerian manifold $(\widetilde{N}, \tilde{h})$ by using a divine structure $\tilde{Q}$ in the form $K=a \widetilde{Q}+b I$, where $a, b \in R^{*}$, thus

$$
K^{2}=a^{2} \tilde{Q}^{2}+2 a b \tilde{Q}+b^{2} I
$$

using that $K^{2}=I$ and $\widetilde{Q}^{2}=\tilde{Q}+I$, we get

$$
\widetilde{Q}_{1}=\frac{I+\sqrt{5} K}{2}, \widetilde{Q}_{2}=\frac{I-\sqrt{5} K}{2} .
$$

Moreover,

$$
\tilde{h}\left(\tilde{Q}_{i}(W), X\right)=\tilde{h}\left(W, \tilde{Q}_{i}(X)\right) \Leftrightarrow \tilde{h}(\tilde{Q}(U), V)=\tilde{h}(U, \tilde{Q}(V))
$$

$\forall i \in\{1,2\}$ and vector fields of tangent $W, X \in \eta(\widetilde{N})$. On a divine Kaehlerian manifold $(\widetilde{N}, \tilde{h}, \tilde{Q})$, we can prescribe two operators' projection

and

$$
\begin{aligned}
& l=\frac{1}{\sqrt{5}}(\Psi I-\tilde{Q}) \\
& m=\frac{1}{\sqrt{5}}(\Psi-I)(I+\tilde{Q})
\end{aligned}
$$




\section{Properties of induced structures on sub-manifolds in $(\widetilde{N}, \widetilde{h}, \widetilde{Q})$}

Let $\mathrm{N}$ be an n-dimensional sub-manifold, absorbed on a Kaehlerian manifold $(\widetilde{N}, \widetilde{h}, \widetilde{Q})$ with a Kaehlerian hermitian matrix $\tilde{h}$ and a divine structure $\tilde{Q}$ such that the matrix $\tilde{h}$ is $\tilde{Q}$ relevant. We denote by $T_{y} N$ the tangent space of $\mathrm{N}$ on $y \in \widetilde{N}$ and by $T_{y}{ }^{\perp} N$ the normal space of $\mathrm{N}$ in $\mathrm{y}$, for every $y \in \widetilde{N}$. Let $i_{*}$ be the immersion differential $i: N \rightarrow \widetilde{N}$.

The induced Kaehlerian hermitian matrix $\mathrm{h}$ of $\mathrm{N}$ is given by

$$
h(W, X)=\tilde{h}\left(i_{*} W, i_{*} Y\right) \text { for all } W, X \in \eta(\tilde{N}) \text {. }
$$

Let us assume an orthonormal basis $\left\{f_{1}, f_{2}, \ldots f_{n}\right\}$ of the normal space $T_{y}(N)^{\perp}$ at each point of $y \in \widetilde{N}$. Let us suppose that range of indices $\mathrm{a}, \mathrm{b}$ and $\mathrm{c}$ are from $1,2 \ldots \mathrm{n}$ and $a, b, c \in\{1, \ldots, n\}$.

For any $W \in T_{y} N, \tilde{Q}_{i_{*}} W, \widetilde{Q} M_{a}$ can be decomposed in normal components and tangential at $\mathrm{N}$ in the form:

$$
\begin{aligned}
& \tilde{Q}_{i_{*}} W=i_{*} Q W+\sum_{a} V_{a}(W) M_{a} \forall W \in \eta(N) \\
& \text { and } \quad \tilde{Q} M_{a}=\varepsilon i_{*} \mu_{a}+\sum_{b} e_{a b} M_{b} \quad(\varepsilon= \pm 1),
\end{aligned}
$$

where $\mathrm{Q}$ is an 1,1 -type tensor field, $\mu_{a}$ are vector fields of tangent on sub-manifold $\mathrm{N}, V_{a}$ are 1-forms on $\mathrm{N}$ and $e:=\left(e_{a b}\right)_{n}$ is a real function $n \times n$ matrix on $\mathrm{N}$. Thus, we get a structure $\left(Q, h, V_{a}, \mu_{a} \cdot\left(e_{a b}\right)_{n}\right)$ inspired on $\mathrm{N}$ by $(\tilde{Q}, \tilde{h})$ from the gauss and Weingarten formulae are:

$$
\begin{aligned}
& \widetilde{\nabla}_{W} X=\nabla_{W} X+\sum_{a=1}^{n} j_{a}(W, X) M_{a} \\
& \widetilde{\nabla}_{W} M_{a}=-B_{a} W+\nabla_{W}^{\perp} M_{a},
\end{aligned}
$$

where

$$
j_{a}(W, X)=h\left(B_{a} W, X\right), \forall W, X \in \eta(N) .
$$

If $\left\{f_{1}, f_{2}, \ldots f_{n}\right\}$ and $\left\{f_{1}^{\prime}, f_{2}^{\prime} \ldots f_{n}^{\prime}\right\}$ are two orthonormal bases on a normal space $T_{y}{ }^{+} N$ then the decomposition of $f_{a}^{\prime}$ in the base $\left\{f_{1}, f_{2}, \ldots f_{n}\right\}$ is the following

$$
F_{a}^{\prime}=\sum_{c=1}^{n} l_{a}^{c} M_{c}
$$

For any $a \in\{1 \ldots n\}$, where $\left(l_{a}{ }^{c}\right)$ is an $n \times n$ orthogonal matrix and we have $V_{a}^{\prime}=\sum_{c} l_{a}^{c} V_{c}$,

$$
\mu_{a}^{\prime}=\sum_{c} l_{a}^{c} \mu_{c} \text { and } e_{a b}^{\prime}=\sum_{c} l_{a}^{c} e_{c d} l_{b}^{d} .
$$

Thus, if $\mu_{1} \ldots \mu_{n}$ are vector fields which is linearly independent, then $\mu_{1}^{\prime} \ldots \mu_{n}^{\prime}$ are also linearly independent further because $e_{a b}$ is symmetric in a and b under a suitable transformation, we can find that $e_{a b}$ can be reduce to $e_{a b}^{\prime}=\lambda_{a} d_{a b}$, where $\lambda_{a}(a \in\{1 \ldots n\})$ are eigen values of the matrix $\left(e_{a b}\right)_{n}$ and in this case we have,

$$
V_{a}^{\prime} \mu_{a}=\varepsilon d_{a b}\left(1+\lambda_{a}-\lambda_{a} \lambda_{b}\right)
$$

and from this we get

$$
V_{a}^{\prime} \mu_{a}=\varepsilon\left(1+\lambda_{a}-\lambda_{a}^{2}\right) V_{a}^{\prime} \mu_{a}=\varepsilon\left(1+\lambda_{a}-\lambda_{a}^{2}\right) .
$$

Proposition 3.1 if $N$ is an n-dimensional sub-manifold of co-dimension $n$, in a divine Kaehlerian manifold $(\widetilde{N}, \widetilde{h}, \widetilde{Q})$, then the structure $\left(Q, h, V_{a}, \varepsilon \mu_{a},\left(e_{a b}\right)_{n}\right)$ induced on $M$ by the structure $\widetilde{Q}$ has the following properties:

$$
\text { i. } \quad\left(\nabla_{W} Q\right)(X)=\mathcal{P}(W, X)+\varepsilon \sum_{a} j_{a}(W, X) \mu_{a}+\sum_{a} V_{a}(X) B_{a} W
$$




$$
\begin{array}{ll}
\text { ii. } & \left(\nabla_{W} V_{a}\right)(X)=\tilde{h}\left(\mathcal{P}(W, X), M_{a}\right)-j_{a}(W, Q X)+\sum_{b}\left(V_{b}(X) m_{a b}(W)+j_{b}(W, X) e_{b a}\right) \\
\text { iii. } & \nabla_{W} \mu_{a}=\mathcal{P}\left(W, M_{a}\right)^{T}-\varepsilon Q\left(B_{a} W\right)+\varepsilon \sum_{b} e_{a b} B_{b} W+\sum_{b} m_{a b}(W) \mu_{b} \\
\text { iv. } & \\
& W\left(e_{a b}\right)=\tilde{h}\left(\mathcal{P}\left(W, M_{a}\right), M_{b}\right)-\varepsilon V_{a}\left(B_{b} W\right)-V_{b}\left(B_{a} W\right)+\sum_{c}\left[m_{a c}(W) e_{c b}+\right. \\
& \left.m_{b c}(W) e_{a c}\right] \\
& \text { for any } W, X \in \eta(N)
\end{array}
$$

Proof: From the equation

$$
\begin{aligned}
\tilde{\nabla}_{W}(\tilde{Q} X)=\nabla_{W} Q X \\
\quad-\sum_{a} V_{a}(X) B_{a} W+\sum_{a}\left[m_{a}(W, Q X)+W\left(V_{a}(X)+\sum_{b} V_{b}(X) m_{b a}(W)\right] M_{a}\right.
\end{aligned}
$$

and

$$
\tilde{Q}\left(\widetilde{\nabla}_{W} X\right)=Q\left(\nabla_{W} X\right)+\varepsilon \Sigma_{a} j_{a}(W, X) \mu_{a}+\Sigma_{a}\left[V_{a}\left(\nabla_{W} X\right)+\Sigma_{b} j_{b}(W, X) e_{b a}\right] M_{a},
$$

we get

$$
\begin{aligned}
& \mathcal{P}(W, X)=\left(\nabla_{W} Q\right)(X)-\sum_{a} V_{a}(X) B_{a} W-\varepsilon \sum_{a} j_{a}(W, X) \mu_{a}+\sum_{a}\left[j_{a}(W, Q X)+\right. \\
& \left.\left(\nabla_{W} V_{a}\right)(X)+\sum_{b} V_{b}(X) m_{b a}(W)-\sum_{b} j_{b}(W, X) e_{b a}\right] M_{a}
\end{aligned}
$$

Thus, the tangential part is identifying and respectively the normal part on the last similarity, we get (i) and (ii) property

$$
\widetilde{\nabla}_{W}\left(\tilde{Q} M_{a}\right)=\varepsilon \nabla_{W} \mu_{a}-\sum_{b} e_{a b} B_{b} W+\sum_{b}\left[W\left(e_{a b}\right)+\varepsilon j_{b}\left(W, \mu_{a}\right)+\sum_{c} e_{a c} \cdot m_{c b}(W)\right] M_{b}
$$

Also, from

$$
\tilde{Q}\left(\widetilde{\nabla}_{W} M_{a}\right)=-Q\left(B_{a} W\right)+\varepsilon \sum_{b} m_{a b}(W) \mu_{b}-\sum_{b}\left[V_{b}\left(B_{a} W\right)-\sum_{c} e_{c b} m_{a c}(W)\right] M_{b},
$$

we get

$$
\begin{aligned}
\mathcal{P}\left(W, M_{a}\right)= & \varepsilon \nabla_{W} \mu_{a}+Q\left(B_{a} W\right)-\varepsilon \sum_{b} m_{a b}(W) \mu_{b} \\
& -\sum_{b} e_{a b} B_{b} W \\
& +\sum_{b}^{b}\left[W\left(e_{a b}\right)+\varepsilon j_{b}\left(W, \mu_{a}\right)+V_{a}\left(B_{a} W\right)\right. \\
& \left.-\sum_{c} e_{c b} m_{a c}(W)-e_{a c} \cdot m_{c b}(W)\right] M_{b}
\end{aligned}
$$

Thus, the tangential part is identifying and respectively the normal part on the last equality, we get (iii) and (iv)

Proposition 3.2 Let $N$ be a $n$-dimensional sub-manifold of co-dimension $n$ in a divine Kaehlerian manifold $(\widetilde{N}, \widetilde{h}, \widetilde{Q})$ with $\tilde{\nabla} \tilde{Q}=0$ if $\left(Q, h, V_{a}, \mu_{a},\left(e_{a b}\right)_{n}\right)$ is the induced structure on $N$ by $(\widetilde{Q}, \tilde{h})$ and $\nabla$ is the levi-civita connection defined on $N$ with respect to $h$ then the Nijenhuis tensor field of $Q$ has the form:

$$
\begin{aligned}
& N_{Q}(W, X)=-\Sigma_{a} h\left(\left(Q B_{a}-B_{a} Q\right)(W), X\right) \mu_{a}-\Sigma_{a} h\left(X, \mu_{a}\right)\left(Q B_{a}-B_{a} Q\right)(W)+ \\
& \sum_{a}\left(W, \mu_{a}\right)\left(Q B_{a}-B_{a} Q\right)(X)
\end{aligned}
$$

For any $W, X \in \eta(N)$. 
Remark: $\mathrm{N}$ be a n-dimensional sub-manifold of co-dimension $\mathrm{m}$ in a divine Kaehlerian manifold $(\widetilde{N}, \widetilde{h}, \widetilde{Q})$ and $\left(Q, h, V_{a}, \mu_{a},\left(e_{a b}\right)_{n}\right)$ be the induced structure on $\mathrm{N}$ by $(\widetilde{Q}, \tilde{h})$ if $\widetilde{\nabla} \tilde{Q}=0$ and $(1,1)$ tensor field $\mathrm{Q}$ on $\mathrm{N}$ commutes with the operators of Weingarten

$$
B_{a}\left(\text { i.e }\left(Q B_{a}=B_{a} Q\right)\right) \text { for any } a \in\{1, \ldots, m\}
$$

then the Nijenhuis tensor field of $\mathrm{Q}$ vanishes on $\mathrm{N}$ i.e., $\left(M_{Q}(W, X)=0\right)$ for any $W, X \in \eta(N)$.

Theorem 3.3 Let $N$ be an n-dimensional non-invariant sub-manifold of co-dimension $m$ immersed in a divine Kaehlerian manifold $(\widetilde{N}, \widetilde{h}, \widetilde{Q})$ so that vector fields of tangential $\eta_{1}, \eta_{2}, \ldots, \eta_{m}$ are linearly independent. Then

$$
\operatorname{trace}(Q)=m-\operatorname{tr}(B)+\sum_{A=m+1}^{n} \lambda_{B}, m<n
$$

and $m-\operatorname{tr}(B), n=m$ with

$$
\lambda_{B} \in\{\Psi,(1-\Psi)\} \forall A, B \in\{m+1, \ldots, n\} .
$$

Proof: Let matrices (Q) (of Q) $V:=\left\{\mu_{1}, \mu_{1}, \ldots, \mu_{m}\right\}$ and $B:=\left(e_{a b}\right)_{m}$.

Let us assume that

$$
(Q) V=V\left(I_{m}-B\right), \text { when } I_{m}=\left(\delta_{a b}\right)
$$

is the identity matrix of order $\mathrm{m}$.

i. For

$$
\begin{aligned}
& m=n, \text { from }|V| \neq 0 \text { we get }(Q) \\
& =V\left(I_{m}-B\right) V^{-1},
\end{aligned}
$$

and from this, we have

$$
Q_{a}^{b}=\sum_{\rho, \sigma} u_{\rho}^{b}\left(\delta_{\sigma}^{\rho}-e_{\sigma}^{\rho}\right) v_{a}^{\sigma}
$$

Here $a, b, \rho, \sigma$ lies from $1,2, \ldots, m$. Also $Q_{a}^{b}, u_{\sigma}^{b}$ and $v_{a}^{\rho}$ are the components of matrices $(Q), V$ and $V^{-1}$ respectively,

hence, we have

$$
\operatorname{tr}(Q)=m-\operatorname{tr}(B) .
$$

ii. For $m<n$

Suppose the matrices

\section{$\bar{V}$ and $O$ by: $\bar{O}$}

$$
\bar{V}=\left(\mu_{1}, \mu_{2}, \ldots, \mu_{m}, \ldots \eta_{m+1} \ldots \eta_{n}\right) \text { and } O=\left(\begin{array}{cc}
\delta_{a b}-e_{a b} & 0 \\
0 & \lambda_{B} \delta_{B A}
\end{array}\right) \text {, }
$$

where

$$
A, B \text { lies from } m+1, \ldots, n A, B \text { lies from } m+1, \ldots, n \text {. }
$$

Here

$$
\delta_{a a}=1, \delta_{a b}=0 \text { for } a \neq b \text { and } \lambda_{B} \in\{\Psi,(1-\Psi)\}
$$

are solutions of the equation

$$
\lambda^{2}=\lambda+1 \lambda^{2}=\lambda+1 \text { for } B \in\{m+1, \ldots, n\} \text { as }|\bar{V}| \neq 0|\bar{V}| \neq 0 \text {. }
$$

Now, from equation

$$
(Q)=\bar{V} O \bar{V}^{-1}(Q)=\bar{V} O \bar{V}^{-1},
$$


we get

$$
Q_{a}^{b}=\sum_{\rho, \sigma} \bar{u}_{\rho}^{b} l_{\sigma}^{\rho} \bar{v}_{a}^{\sigma}(a, b, \rho, \sigma) \in\{1,2, \ldots, m\},
$$

where $Q_{a}^{b}, \bar{u}_{\rho}^{b}, l_{\sigma}^{\rho}$ and $\bar{v}_{a}^{\sigma}$ respectively are the components of matrices $(Q), \bar{V}, O$ and $\bar{V}^{-1}$.

Hence, we have

$$
\operatorname{tr}(Q)=m-\operatorname{tr}(B)+\sum_{A=m+1}^{n} \lambda_{B} .
$$

Proposition 3.4 Let $N$ be an n-dimension sub-manifold and $m$ be a co-dimension in a divine Kaehlerian manifold $(\widetilde{N}, \widetilde{h}, \tilde{Q})$ and let $\left(Q, h, V_{a}, \mu_{a},\left(e_{a b}\right)_{n}\right)$ be the induced structure in $N$ by $(\tilde{h}, \tilde{Q})$, then $N$ is an inspired structure $(\tilde{h}, \tilde{Q})$ which is invariant on $N$ is a divine Kaehlerian structure, whenever $Q$ is nontrivial.

Proof: If $\mathrm{N}$ is an invariant sub-manifold in a divine Kaehlerian structure $(\widetilde{N}, \widetilde{h}, \widetilde{Q})$ the $(\widetilde{Q}, \tilde{h})$ is a divine Kaehlerian manifold

Conversely, if we assume that $(\widetilde{N}, \widetilde{h}, \widetilde{Q})$ is a divine Kaehlerian structure then

$$
\Sigma_{a}\left(u_{a}(Y)\right)^{2}=0
$$

and we get

$$
\sum_{a} u_{a}(Y) h\left(Y, u_{a}\right)=\sum_{a}\left(u_{a}(Y)\right)^{2}=0
$$

where $u_{a}(Y)=0$ for $a \in(1,2, \ldots, m)$ and hence $\mathrm{N}$ is invariant.

\section{Example: -}

We see that the vast space is a $(m+n)$-dimensional Euclidian space $F^{m+n}\left(m, n \in M^{*}\right)$.

Let $\tilde{Q}: F^{m+n} \rightarrow F^{m+n}$ be a $1,1-$ tensor field prescribed by

$$
\begin{aligned}
& \tilde{Q}\left(x^{1}, \ldots, x^{m}, y^{1}, \ldots, y^{n}\right) \\
& \quad=\left(\Psi x^{1}, \ldots, \Psi_{x^{m}},(\Psi-1) y^{1}, \ldots,(\Psi-1) y^{n}\right)
\end{aligned}
$$

For each point

$$
\left(x^{1}, \ldots, x^{m}, y^{1}, \ldots, y^{n}\right) \in F^{m+n} \text { where } \Psi=\frac{1+\sqrt{5}}{2} \text { and } 1-\Psi=\frac{1-\sqrt{5}}{2}
$$

are the real roots of the equation $y^{2}=y+1$.

On the other side

$$
\text { for }\left(x^{1}, \ldots, x^{m}, y^{1}, \ldots, y^{n}\right),\left(u^{1}, \ldots, u^{m}, v^{1}, \ldots, v^{n}\right) \in F^{m+n} \text {, }
$$

we have

$$
\begin{aligned}
& \widetilde{Q}^{2}\left(x^{1}, \ldots, x^{m}, y^{1}, \ldots, y^{n}\right)=\left(\Psi^{2} x^{1}, \ldots, \Psi^{2} x^{m},(1-\Psi)^{2} y^{1}, \ldots,(1-\Psi)^{2} y^{2}\right) \\
& =\left(\Psi x^{1}, \ldots, \Psi x^{m},(1-\Psi) y^{1}, \ldots,(1-\Psi) y^{n}+\left(x^{1}, \ldots, x^{m}, y^{1}, \ldots, y^{n}\right)\right.
\end{aligned}
$$

Thus, we get $\widetilde{Q}^{2}=\tilde{Q}+I$

and

$$
\begin{aligned}
<\tilde{Q}\left(x^{1}, \ldots, x^{m}, y^{1}, \ldots, y^{n}\right),\left(u^{1}, \ldots, u^{m}, v^{1}, \ldots, v^{n}\right)> \\
=<\left(x^{1}, \ldots, x^{m}, y^{1}, \ldots, y^{n}\right), \tilde{Q}\left(u^{1}, \ldots, u^{m}, v^{1}, \ldots, v^{n}\right)>
\end{aligned}
$$

for every point $\left(x^{1}, \ldots, x^{m}, y^{1}, \ldots, y^{n}\right),\left(u^{1}, \ldots, u^{m}, v^{1}, \ldots, v^{n}\right) \in F^{m+n}$

Therefore, the scalar product on $F^{m+n}$ is relevant to $\widetilde{Q}$ is the divine structure on $F^{m+n},<>$ and $\left(F^{m+n},<>, \tilde{Q}\right)$ and hence it represents the divine Kaehlerian manifold. 


\section{Conclusion}

Here is the brief discussion over some crucial results of this article:

Section 1 is introductory one, that includes the basic concepts related to divine Kaehlerian manifold which is Riemann manifold of even dimension, and whose holonomy group is contained in the unitary group.

In section 2, the property of divine Kaehlerian manifold in terms of Fibonacci sequence, trace and Eigen values of the divine structure in a Kaehlerian manifold has been studied. Furthermore, it is investigated that each 'almost product structure' in an n- dimensional Kaehlerian manifold induced the two divine structures.

In section 3, properties of the structure induce other structures and linearly independent existence for n-dimensional sub-manifolds in a Kaehlerian manifolds were studied. The inspired structured in a divine Kaehlerian manifold and induced structure in terms of non-trivial invariant have also been investigated.

\section{References}

Bayram, S and Mehmet, A. A. (2014). Golden maps between Golden Riemannian Manifolds and Constancy of certain maps, Mathem. Сотти.19 333-342.

Crasmareanu, M., Hretcanu, C. E. (2007). On some invariant submanifolds in a Riemannian manifold with golden structure, Analele Stintifice Universitatis" Al. I. Cuza" DIN IASI (S.N.) Matematica, Tomul LIII, pp 199211.

Etayo, F., Santamaria, R., Upadhyay, A. (2017). On the geometry of almost golden Riemannian manifolds, Mediterr. J. Math. 187(14) 991--1007.

Gheorge, P. (1986). On some sub-manifolds of a locally product Riemannian manifold, Kodai math. J. No. 9, 327-333.

Ghyka, M. (1977). The geometry of art and life, Dover publications, 2 Edition, pp 7-19.

Goldberg, S. I. and Petridis, N. C. (1973). Differentiable solutions of algebraic equations on manifolds, Kodai Math Sem. Rep. No. 25,111-128.

Goldberg, S. I. and Yano, K. (1970). Polynomial structures on manifolds, Kodai Math. Sem. Rep., No. 22, 199-218.

Hretcanu, C. E. (2007). Submanifolds in Riemannian Manifold with golden structure, Workshop on Finsler geometry and its applications, Hungary.

Hretcanu, C. E. and Crasmareanu, M. C. (2009). Applications of the golden ratio on Riemannian Manifolds, Turk J Math, 33, 179-191.

http:/len. Wikipedia-org/wiki/golden ratio

Kumar, S. and Petwal, K. C. (2011). Functioning of Divine Ratio on Conformal Einstein Spaces, British J Math. Comp. Sci, 1(2), pp 87-100.

Sarma, S. and Bhuyar, R. (2018). Fibonacci number, Golden ratio and their connection to different floras, Int. J. Math. Trends and Tech., 61(2) 95-99.

Tyuzi, A. (1981). Sub-manifolds of an almost product Riemannian manifold, Kodai math. J. 4 327-343.

Yano, K. and Kon, M. (1984). Structures on Manifolds, Series in Pure Mathematics Volume 3, World Sci. Publishing Co., Singapore.

$* * * * * * *$ 